\title{
Auto-associative Neural Networks to Improve the Accuracy of Estimation Models
}

\author{
Salvatore A. Sarcia ${ }^{\text {, }}$, Giovanni Cantone ${ }^{1}$ and Victor R. Basili ${ }^{2,3}$
}

${ }^{1}$ DISP, Università di Roma Tor Vergata, via del Politecnico 1, 00133 Rome, Italy

${ }^{2}$ Dept. of Computer Science, University of Maryland, A.V. Williams Bldg. 115, 20742, College Park, Maryland, USA

${ }^{3}$ Fraunhofer Center for Experimental Software Engineering Maryland, 20742, College Park, Maryland, USA

\section{KEY TERMS}

PREDICTION MODELS, ESTIMATION MODELS, CURVILINEAR COMPONENT ANALYSIS, NON-LINEAR PRINCIPAL COMPONENT ANALYSIS, FEATURE REDUCTION, FEATURE SELECTION, NEURAL NETWORKS, AUTO-ASSOCIATIVE NEURAL NETWORKS, COMPUTATIONAL INTELLIGENCE, SOFTWARE ESTIMATION, SOFTWARE ECONOMICS, SOFTWARE ENGINEERING.

\section{ABSTRACT \\ Prediction of software engineering variables with high accuracy is still an open problem. The primary reason for the lack of high accuracy in prediction might be because most models are linear in the parameters and so are not sufficiently flexible and suffer from redundancy. In this chapter, we focus on improving regression models by decreasing their redundancy and increasing their parsimony, i.e., we turn the model into a model with fewer variables than the former. We present an empirical auto-associative neural network-based strategy for model improvement, which implements a reduction technique called Curvilinear component analysis. The contribution of this chapter is to show how multi-layer feedforward neural networks can be a useful and practical mechanism for improving software engineering estimation models. \\ INTRODUCTION \\ Prediction of software engineering variables such as project cost, fault proneness, and number of defects is a critical issue for software organizations. It is important to get the best estimate possible when planning a new project, activity, or task. For instance, we may have to predict the software cost, the effort in carrying out an activity (e.g. coding a module), or the expected number of defects arising from a module or sub-system. Improving the prediction capability of software organizations is one way of improving their competitive advantage. Better predictions can improve the development process in terms of planning resources, setting and achieving quality goals, and making more informed decisions about the schedule. The point is that, prediction is a major problem when trying to better manage resources, mitigate project risk, and}


deliver products on time, on budget and with the required features and functions (quality) (Boehm, 1981).

Despite the fact that prediction of software variables with high accuracy is a very important issue for competing software organizations, it is still an unsolved problem (Shepperd, 2007). A large number of different prediction models have been proposed over the last three decades (Shepperd, 2007). There are predefined mathematical functions (e.g., FP-based functions, COCOMO-I, and COCOMO-II), calibrated functions (e.g., function based on a regression analysis on local data), machines learning (e.g., estimation by analogy, classification and regression trees, artificial neural networks), and human-based judgment models. There exist a large number of empirical studies aimed at predicting some software engineering variable (Myrtveit, 2005). Most often, the variable is software project cost, which we will use as the main exemplar of the approach described in this chapter.

In this chapter, we present a computational intelligence technique to improve the accuracy of parametric estimation models based on regression functions. The improvement technique is defined, tested, and verified through software cost estimation data (Sarcia', Cantone, \& Basili, 2008). In particular, this chapter offers a technique that is able to improve the accuracy of loglinear regression models in the context of the COCOMO-81 projects (Boehm, 1981; Shirabad, \& Menzies, 2005). From a mathematical point of view, even if we change the model variables, e.g., we predict effort instead of predicting the number of defects, or vice versa, the strategy to improve the estimation model does not change at all. This means that we can apply the improvement strategy to any set of variables in any kind of development context without change. Of course, it does not mean that the improvement technique will succeed in every context.

It is important to note that, even if we were making predictions through human-based judgment, to increase estimation accuracy we would have to build parametric models calibrated to the local data and use them to estimate variables of interest for the specific context of the organization (McConnell, 2006). For instance, productivity (lines of code/time) may change according to the context of the organization (e.g., capability of analysts and developers, complexity of the software system, environment of the development process). The point is that, even experts should use regression functions for gathering suitable information from the context in which they are operating. Therefore, apart from the kind of estimation model used, when improving estimation accuracy, calibrating regression functions is a required activity. We believe that, enhancing accuracy of regression functions is the core of any estimation activity. That is why we focus upon improving regression functions and do not deal with other approaches.

We begin with an introduction that provides the general perspective. Then, we provide some background notes, required terminology, and define the problem. We present, from a practical point of view, the main issues concerning parametric estimation models, multi-layer feedforward neural networks, and auto-associative neural networks. We illustrate the empirical strategy for dealing with the problem (the solution). We conclude with a discussion of the benefits and drawbacks of the methodology and provide some ideas on future research directions. 


\section{PARAMETRIC ESTIMATION MODELS}

The estimation models that we refer to are based on parametric models as illustrated in Figure 1.

Figure 1. Parametric estimation model (regression functions).

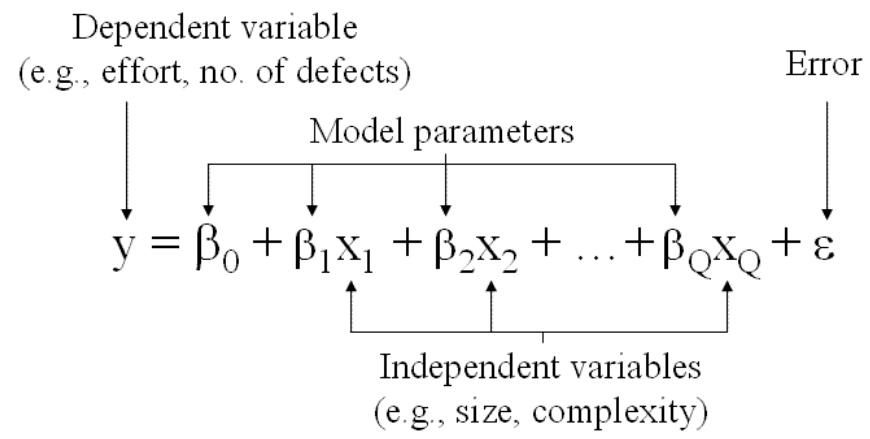

Mathematically, the estimation model (EM) in Figure 1 can be represented by a regression function $f_{R}$ such that $y=f_{R}(x, \beta)+\varepsilon$, where $x$ is a set of independent variables, $y$ is the dependent variable, and $\beta=\left(\beta_{0} \ldots \beta_{Q}\right)$ is a set of parameters defining $f_{R}$. The component $\varepsilon$ is the aleatory part of the model (i.e. the unknown part depending on the probability of future events) representing our uncertainty about the relationship between the independent and dependent variables, with $E(\varepsilon)=0$ and $\operatorname{cov}(\varepsilon)=\sigma^{2} I$ (Weisberg, 1985). To calculate function $f_{R}$, we need to know every point of the population. This almost never happens. Then, since we know only a finite sample from the population, we can only estimate $\beta$ by finding a set of estimators $B=\left(b_{0}\right.$ $\left.\ldots b_{Q}\right)$ such that they minimize an error function, e.g., the least squares error function. To estimate $\mathrm{B}$, we use previous observations of both dependent $(\mathrm{Y})$ and independent $(\mathrm{X})$ variables (Figure 2).

Figure 2. Least squares estimate

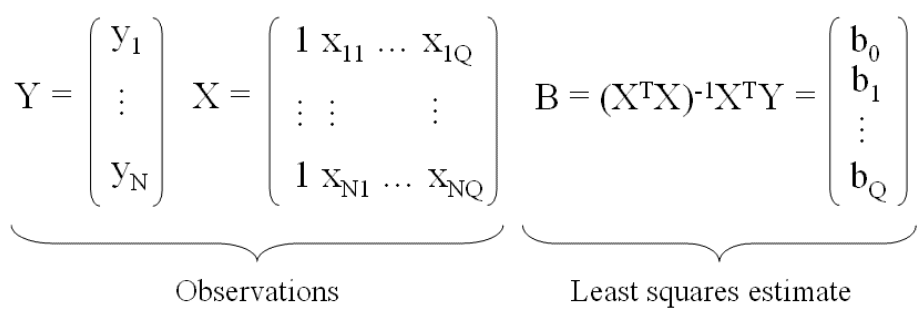

We consider the relationship $Y=f_{R}(X, B)$, where $Y$ is a set of observations of the dependent variable, $\mathrm{X}$ is the observation matrix including $\mathrm{N}$ observations of $\mathrm{Q}$ independent variables, and $\mathrm{B}$ is the vector of parameters estimating $\beta$ (Figure 2). Note that, we use a lowercase letter (e.g., $x$ ) to refer to the variables and an uppercase letter (e.g., $\mathrm{X}$ ) to refer to the data. Because of $\varepsilon$, the least squares estimate, i.e. $B$ in Figure 2, are different from parameters $\beta$. Thus, $f_{R}$ fed with $X$ provides $Y_{\text {est }}=f_{R}(X, B)$, not $Y$. Then, the difference $e=Y-Y_{\text {est }}$ is a vector of errors representing $\varepsilon$ (called residuals, with $\mathrm{e} \neq \varepsilon$ ). The most important part in modeling is to find the best estimates for $\beta$. This activity is called parameter estimation, fitting, or calibration. Note that, the LS estimate in Figure 2 is based on finding the minimum of the sum of squares of the error. 
From a practical point of view, the minimum of the sum of squares of the error can be found by setting the gradient to zero, where the derivative is made with respect to each parameter. Therefore, minimizing the cost function means solving a system of equations of partial derivatives set to zero (McQuarrie \& Tsai, 1998, Pedhazur, 1997). If the system of equations is composed of a linear combination of the sought parameters, the EM is linear in the parameters, and we have a closed solution (B in Figure 2). If the system of equations is composed of nonlinear equations of the sought parameters, the EM is non-linear in the parameters and the solution can be found iteratively (there is no closed solution).

Note that, even a non-linear polynomial such as $y=\beta_{0}+\beta_{1} x_{1}+\beta_{2} x_{2}+\beta_{3} x_{1} x_{2}+\beta_{4} x_{1}{ }^{2}+\beta_{5} x_{2}{ }^{2}$ is linear in the parameters. This can be obtained by transforming its variables as follows $\mathrm{x}_{1} \mathrm{x}_{2}=\mathrm{x}_{3}$, $x_{1}{ }^{2}=x_{4}$, and $x_{2}{ }^{2}=x_{5}$. Therefore, $y=\beta_{0}+\beta_{1} x_{1}+\beta_{2} x_{2}+\beta_{3} x_{3}+\beta_{4} x_{4}+\beta_{5} x_{5}$, which is still a linear model where we can apply the least squares estimate in Figure 2. Another important transformation is about turning a geometric model into a linear model through logarithms. The resulting function is called log-linear regression function. For instance, $y=x_{1}{ }^{a} \prod_{i=1 . . k} c_{i}$ is a geometric model having a variable $\mathrm{x}_{1}$, a coefficient " $\mathrm{a}$ " as a power of $\mathrm{x}_{1}$, and $\mathrm{k}$ multiplicative coefficients. Taking the logarithm of both sides of $y=x_{1}{ }^{a} \prod_{i=1 . . k} c_{i}$, we can break down the product into sums. Based on the logarithm properties, i.e. $\ln (p q)=\ln (p)+\ln (q)$ and $\ln \left(p^{q}\right)=(q) \ln (p)$, we can write $\ln (\mathrm{y})=\beta_{1} \ln \left(\mathrm{x}_{1}\right)+\beta_{2} \ln \left(\mathrm{c}_{1}\right)+\ldots+\beta_{\mathrm{k}+1} \ln \left(\mathrm{c}_{\mathrm{k}}\right)$, where we have used the letter $\beta$ to indicate the model parameters (with $\mathrm{a}=\beta_{1}$ ). Then, we can set $\mathrm{z}=\ln (\mathrm{y}), \mathrm{h}_{1}=\ln \left(\mathrm{x}_{1}\right), \mathrm{h}_{2}=\ln \left(\mathrm{c}_{1}\right) \ldots \mathrm{h}_{\mathrm{k}+1}=$ $\ln \left(c_{k}\right)$ and add an intercept $\left(\beta_{0}\right)$ so that the log-linear model becomes $z=\beta_{0}+\beta_{1} h_{1}+\beta_{2} h_{2}+\ldots+$ $\beta_{\mathrm{k}+1} \mathrm{~h}_{\mathrm{k}+1}$, which is still a model linear in its parameters where we can use the least squares estimate in Figure 2. Note that, we use an intercept $\left(\beta_{0}\right)$ because if the remaining monomials (i.e., $\beta_{\mathrm{i}} \mathrm{h}_{\mathrm{i}}$, with $\mathrm{i}=1$ to $\mathrm{k}+1$ ) vanish (in case of non-correlation), the equation $\mathrm{z}=\beta_{0}+\beta_{1} \mathrm{~h}_{1}+\beta_{2} \mathrm{~h}_{2}+\ldots+$ $\beta_{\mathrm{k}+1} \mathrm{~h}_{\mathrm{k}+1}$ continues to be satisfied $\left(\mathrm{z}=\beta_{0}\right)$.

Note that, if we want to get the best linear unbiased estimators (BLUE) of $\beta$ (Gauss-Markov theorem) and use the model for inference, LS requires some assumptions (Pedhazur, 1997):

- Errors $\varepsilon$ are not x correlated.

- The variance of the errors is constant (homoscedasticity), $\operatorname{cov}(\varepsilon)=\sigma 2 \mathrm{I}$.

- Errors $\varepsilon$ are not auto-correlated.

- The probability density of the error is Gaussian, $\varepsilon \sim \mathrm{NID}(0, \sigma 2 \mathrm{I})$, i.e. there are no outliers, skewed/kurtotic distributions, and measurement error.

Since the characteristic expressing the relationship between inputs and output is called model shape, the equation in Figure 1 has a linear shape and is linear in the parameters. For instance, a third degree polynomial has a cubic shape and is still linear in the parameters. Complexity of a model is another characteristic expressing the number of parameters composing the model. The more parameters the model has, the more flexible the model is. Models that are linear in the parameters have as many parameters as variables (i.e., one and only one parameter for each variable). Therefore, to increase the model flexibility (i.e. the number of parameters) of a linearin-the-parameter model we have to increase its variables (e.g., increasing the polynomial degree). Barron (1993) proves that the number of parameters required to execute an approximation with a fixed accuracy increases exponentially with the number of variables for models that are linear in the parameters, while it increases linearly with the number of variables for models that are nonlinear in the parameters. This means that, to take advantage fully of parsimony we should consider non-linear-in-the-parameter models instead of the linear ones. If we use models that are non-linear in their parameters such as multi-layer feed-forward neural networks, we can increase 
the model flexibility (i.e. increasing the number of parameters) without adding new variables. This is the real essence of the parsimony (Dreyfus, 2005, pp. 13-14).

However, as mentioned above, non-linear models do not have a closed solution for estimating parameters B. Therefore, to overcome this problem, researchers and practitioners often prefer to use models that are linear in the parameters instead of using the non-linear ones. However, if we choose models that are linear in the parameters, finding the model shape that best fits the data is much more difficult than if we used models that are non-linear in the parameters (Dreyfus, 2005, pp. 136-138). Conversely, iterative procedures require a lot of experience to be effectively used in practical problems. For instance, iterative procedures may provide different parameter estimates depending on the initialization values (Bishop, 1995, pp. 140-148; Dreyfus, 2005, pp. 118-119). Therefore, finding the right parameters may require many attempts as well as the use of optimization techniques to reduce the calibration time.

\section{Estimation Model Accuracy}

Prediction models may be evaluated in terms of Absolute Error (AE), i.e. AE = Actual Estimated $=\mathrm{Y}-\mathrm{Y}_{\text {est }}$. However, an absolute error measure makes no sense when dealing with estimation model accuracy for software engineering variables (e.g., effort, defects). In fact, an Absolute Error would increase with the size of what we we're predicting. Let $\mathrm{AE}_{1}(=0.30)$ and $\mathrm{AE}_{2}(=0.25)$ be the absolute errors calculated by feeding the estimation model with data from two different objects (e.g. project 1 and 2). Then, we might argue that the model is less accurate for project 1 than for project 2 because $A_{1}$ expresses a lower accuracy than $A E_{2}$, i.e., $A E_{1}>$ $\mathrm{AE}_{2}$. However, if $\mathrm{Y}_{1}$ (the actual value from which $\mathrm{AE}_{1}$ has been calculated) were much greater than $\mathrm{Y}_{2}$, i.e., $\mathrm{Y}_{1}>\mathrm{Y}_{2}$, and $\mathrm{AE}_{1} \approx \mathrm{AE}_{2}$ or even $\mathrm{AE}_{1}>\mathrm{AE}_{2}$, then looking at the absolute error $\mathrm{AE}_{1}$, we would incorrectly judge that the estimation model accuracy on object 1 is worse than the accuracy on object 2 . Actually, we should judge the accuracy on object 1 as better than the accuracy on object 2 , i.e. the (absolute) error should be evaluated with respect to the magnitude of the predictions. For instance, it should be much more severe having a prediction error $\mathrm{AE}=$ 0.30 for a project of $100 \mathrm{man} / \mathrm{months}$ than a prediction error $\mathrm{AE}=0.30$ for a project of 10,000 $\mathrm{man} / \mathrm{months}$. This means that, an absolute measure should not be used for model comparison. When predicting software engineering variables, the right choice is to consider a relative error measure, i.e. a measure that takes into account the size of what we are predicting (Myrtveit, Stensrud, \& Shepperd, 2005). For this reason, Boehm (1981) defined the performance of his software cost model (COnstructive COst MOdel, COCOMO) in terms of Relative Error (RE), Eqn. 1.

$$
\mathrm{RE}=(\text { Actual }- \text { Estimated }) / \text { Actual }=\left(\mathrm{Y}-\mathrm{Y}_{\mathrm{est}}\right) / \mathrm{Y} .
$$

Note that, currently the Boehm's model has enhanced into the COCOMO-II (Boehm, Horowitz, Madachy, Reifer, Clark, Steece, Brown, Chulani, \& Abts, 2000), but the accuracy evaluation principles have not changed. The evaluation procedure of the model accuracy is shown in Figure 3. 
Figure 3. Evaluating estimation models

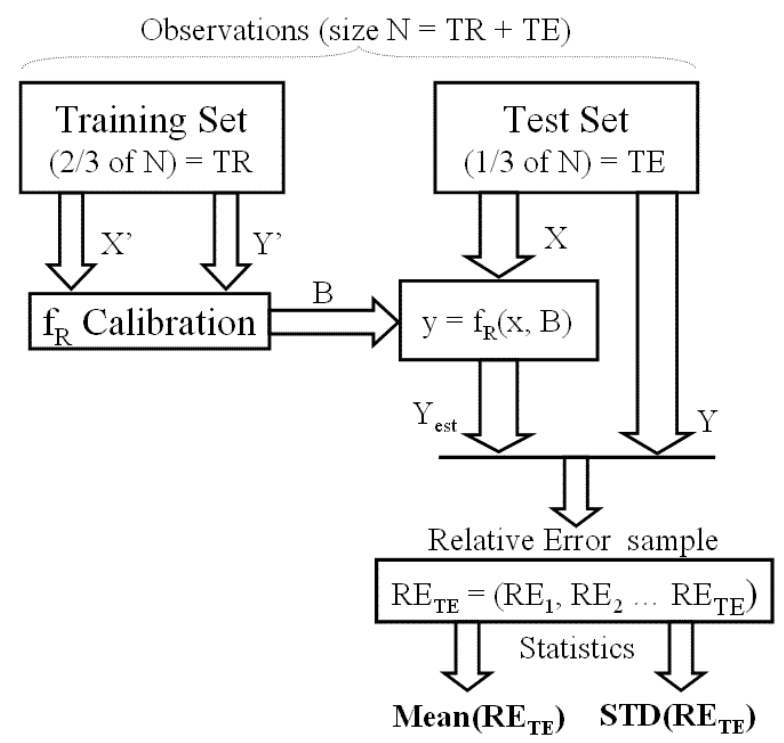

We start with considering a data set of size $\mathrm{N}$, which is split up into two subsets, a training set and a test set. The training set size is $\mathrm{TR}=(2 / 3) \mathrm{N}$ and the test set size is $\mathrm{TE}=(1 / 3) \mathrm{N}$, thus $\mathrm{N}=$ $\mathrm{TR}+\mathrm{TE}$. We choose the splitting proportion 2/3-1/3 because it is a usual choice when dealing with hold-out methodologies (Chen, Mezies, Port, \& Boehm, 2005; Kohavi \& John, 1997). A further valid proportion is $80 \%-20 \%$ (Dreyfus, 2005, pp. 103-106). Based on the training set, we calibrate function $f_{R}$, i.e., estimate parameters $B$ by the least squares estimates (Figure 2). Note that, to avoid any confusion between the training set and the test set, we use an apostrophe to point out that the data belongs to the training set (i.e., $X^{\prime}$ and $\left.Y^{\prime}\right)$. Moreover, it is important noting that, differently from X' (Figure 3), the test set X (Figure 3) does not have a vector of 1s in the first column. Once we have calculated the parameters $B$, we feed $X$ into $f_{R}$ and obtain the estimated values $Y_{\text {est }}$. Notice that, the size of $Y_{\text {est }}$ is TE, i.e. $Y_{\text {est }}=\left(Y_{\text {est }}{ }^{(1)} \ldots Y_{\text {est }}{ }^{(T E)}\right)$. Then, accuracy is evaluated by calculating two summary statistics over the Relative Error sample as shown in Figure 3. In particular, Mean $\left(R E_{T E}\right)=(1 / T E) \sum_{i=1 . . T E}\left(R E_{i}\right)$ is a measure of the bias of $f_{R}$ and $\operatorname{STD}\left(\mathrm{RE}_{\mathrm{TE}}\right)=\operatorname{RTSQ}\left\{(1 / \mathrm{N}) \sum_{\mathrm{i}=1 . . \mathrm{TE}}\left(\left[\mathrm{RE}_{\mathrm{i}}-\operatorname{Mean}\left(\mathrm{RE}_{\mathrm{TE}}\right)\right]^{2}\right)\right\}$ is a measure of the spread of $\mathrm{f}_{\mathrm{R}}$. Note that, a correct evaluation can be done through both statistics at the same time. For instance, if we find out that a model has a Mean $\left(\mathrm{RE}_{\mathrm{TE}}\right)$ closer to zero with respect to another model, we can infer that the former is more accurate than the latter in terms of correctness. Moreover, if the former has a narrower $\operatorname{STR}\left(\mathrm{RE}_{\mathrm{TE}}\right)$ than the latter, we can infer that the former is more accurate in terms of reliability (stability).

It is very important to note that, sometimes parametric models are evaluated in terms of Magnitude of Relative Error (MRE) (Conte, Dunsmore, \& Shen, 1986; Shirabad \& Mezies, 2005), where $M R E_{i}=\operatorname{abs}\left(R E_{i}\right)$. The evaluation is performed by two summary statistics, as well. The first one is MMRE $=(1 / \mathrm{TE}) \Sigma_{\mathrm{i}=1 . . \mathrm{TE}}\left(\mathrm{MRE}_{\mathrm{i}}\right)$ and the second one is $\operatorname{PRED}(\mathrm{H})$, Eqn. (2).

$$
\operatorname{PRED}(\mathrm{K})=(100 / \mathrm{TE}) \sum_{\mathrm{i}=1 . . \mathrm{TE}}\left\{\begin{array}{l}
1 \rightarrow \mathrm{MRE}_{\mathrm{i}} \leq(\mathrm{K} / 100) \\
0 \rightarrow \text { otherwise }
\end{array}\right.
$$


$\operatorname{A~PRED}(25)=80 \%$ means that $80 \%$ of the estimates have an error not greater than 0.25 . Since when calculating the MMRE, differently from Mean $\left(\mathrm{RE}_{\mathrm{TE}}\right)$, positive errors and negative errors do not cancel reciprocally, MMRE would seem a better statistic than Mean $\left(\mathrm{RE}_{\mathrm{TE}}\right)$. However, Kitchenham et al. (2001) prove that MMRE and $\operatorname{PRED}(\mathrm{K})$ cannot be used for comparing estimation model accuracy because neither MMRE nor PRED $(\mathrm{H})$ express bias or spread of the model. In particular, they show that MMRE and PRED(N) measure spread and kurtosis of the random variable $\mathrm{Z}=$ Estimated/Actual, respectively. Nevertheless, MMRE may be a useful measure when evaluating the goodness-of-fit of a model.

\section{THE PROBLEM}

The problem that we deal with in this chapter is to improve (parametric) models that are linear in the parameters. There are many ways of improving regression model accuracy. For instance, we can (1) choose a better function, e.g. the one that has a more suitable shape or (2) select input variables that are more relevant. In the former, the game is to select a function shape that best fits the data. In the latter, the real problem is that, once we have selected the complete input variable set, we need to remove redundancy that negatively affects the performance of the estimation model (e.g., by taking out irrelevant variables).

As reported in the Section "Parametric Estimation Models", however, practitioners and researchers prefer using models that are linear in the parameters because they have a closed solution to the problem of calculating the model parameters (Figure 2), while models that are non-linear in the parameters do not. Then, to support those practitioners and researchers in their practical problems the best that we can do is to improve regression models that they actually use, i.e. the linear-in-the-parameter ones. That is why, we focus on removing the redundancy of linear models, and we do not consider non-linear models as a way of improving estimation accuracy.

Taking out redundancy is about finding a model that is more parsimonious (i.e., the one having fewer input variables). Such a model is preferable because it is able to provide better estimates with the same number of observations. Let $\mathrm{N}$ be this number. If we use a function having fewer variables, we can get further degrees of freedom to calibrate its parameters. Vice versa, since $\mathrm{N}$ is constant, if we consider functions having additional variables, the degrees of freedom in calibrating the model parameters decrease and the estimates will be less accurate (Barron, 1993; Dreyfus, 2005, p. 14). Therefore, parsimony can be considered as a power multiplier for regression functions. Intuitively, the rationale of this power enhancement is the same as the example of a plane calculation. We cannot define a plane using only two points because a plane has three parameters, i.e. we need at least three points to determine the equation of a plane. Therefore, since the data set size $(\mathrm{N})$ stays constant, if we reduce the number of input variables (i.e., making the model more parsimonious), we can increase the accuracy of the model.

To increase parsimony of a regression function we have substantially two options. We can (1) shrink the input set into an equivalent pattern or (2) remove irrelevant variables. The former is usually performed by applying Principal Component Analysis (PCA) (Jollife, 1986). The latter is based on methods where the least irrelevant variables are taken out stepwise (Chen, Mezies, Port, \& Boehm, 2005; Kohavi \& John, 1997). Actually, there would be another option to make the model more parsimonious, but it is generally inapplicable because of its too high computational cost. This technique is the exhaustive procedure. It consists of considering all the combinations that we can get with $\mathrm{Q}$ elements. For instance, if we have $\mathrm{Q}$ variables, we have to consider $2^{\mathrm{Q}}$ different combinations, i.e. $2^{\mathrm{Q}}$ models, each having different variables from each other. The basis 
of the power is 2 because an element can belong to the set or not (binary choice). For each model, the procedure goes on by calculating the error. Then, the model having the highest accuracy is selected.

In this work, we present an empirical strategy to shrink the input set by applying an improved version of PCA called Curvilinear Component Analysis (CCA) or non-linear Principal Component Analysis (Sarcia', Cantone, \& Basili, 2008). We apply CCA instead of using PCA because CCA is able to overcome some drawbacks affecting PCA. For instance, PCA can just find linear redundancy, while CCA can find both linear and non-linear redundancy. Note that, to increase the model parsimony we focus on input reduction techniques instead of the stepwise ones because the former can be completely automated while the latter cannot. In fact, stepwise techniques require making a decision as to variables to be removed, i.e. the least relevant variables. If we do not know the relevance of each variable, stepwise techniques may be difficult to be correctly applied.

\section{CURVILINEAR COMPONENT ANALYSIS}

CCA is a procedure for feature reduction that consists of turning a training set into a more compact and equivalent representation. CCA is a compression technique that maps a set into another set having fewer variables, where the mapping is non-linear. We implement CCA through auto-associative multi-layer feed-forward neural networks (Bishop, 1995, pp. 314-319). Implementing CCA does not require being an expert in neural network (NN). A CCA implementation can be found in any mathematical application that is able to deal with NNs and/or matrices. CCA requires just a few lines of code. Even if one does not have the opportunity to use a mathematical suite, a CCA algorithm can be easily implemented through the same programming language used for estimating the LS parameters. Before delving into details of auto-associative neural networks, we provide some background on multi-layer feed-forward neural networks.

\section{Multi-layer Feed-forward Neural Networks}

A neuron is a function $\mathrm{y}=\theta\left(\mathrm{w}_{0}+\Sigma_{\mathrm{i}=1 . . \mathrm{Q}} \mathrm{w}_{\mathrm{i}} \mathrm{X}_{\mathrm{i}}\right)$, where $\theta$ can be linear or non-linear (Figure 4). If $\theta$ is linear (i.e., is the identity function), the neuron is called linear neuron and is the same as a polynomial of first degree. In other words, a linear polynomial is a particular case of a linear neuron. If $\theta$ is non-linear, the neuron is called non-linear, e.g., $\theta$ can be a sigmoid function such as the logistic function or the hyperbolic tangent function (Dreyfus, 2005, pp. 2-3). 
Figure 4. Representation of a neuron

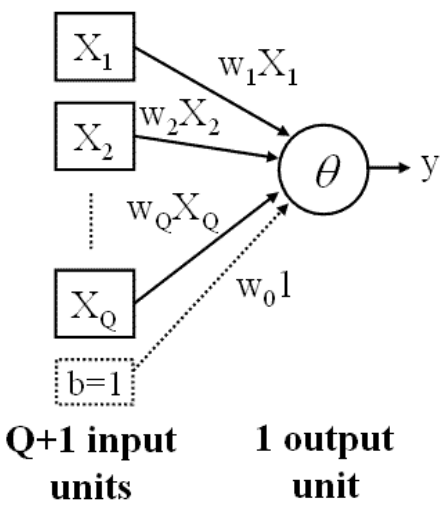

A neuron (also called unit) calculates an output (y) by summing the products between a weight $\mathrm{w}_{\mathrm{i}}$ and an input $\mathrm{X}_{\mathrm{i}}$, with $\mathrm{i}=1$ to $\mathrm{Q}$. Note that, weights are called parameters, as well. Function $\theta$ is called activation function. The input labeled " $\mathrm{b}=1$ " is called bias. It is an input that provides a constant value of 1 . The bias unit plays the same role as the intercept in a polynomial.

Figure 5. A multi-layer feed-forward neural network.

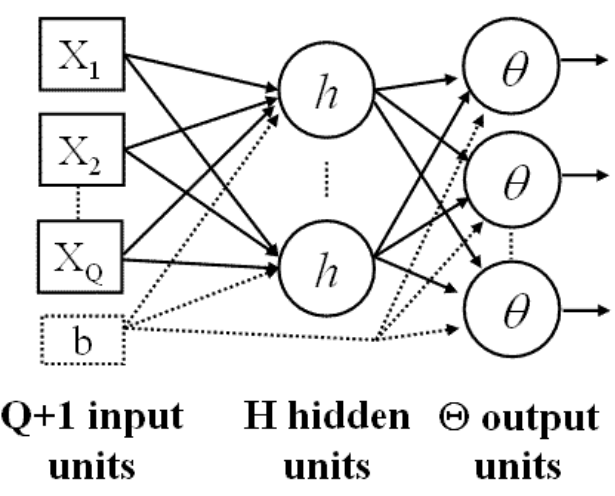

A Multi-layer Feed-forward Neural Network (MFNN) is generally composed of non-linear neurons arranged on some layers (Figure 5). In a feed-forward network, data can only flow from the inputs toward the outputs. In recurrent networks, the data can flow backward, as well. In Figure 5, $h$ may be different from $\theta$. For instance, in regression problems, $h$ is non-linear and $\theta$ is linear (the identity function). In discrimination problems, both $h$ and $\theta$ are non-linear. In Figure 5 , units in the middle (labeled $h$ ) are called hidden because they are hidden from the outside. Note that, the hidden layer expresses the complexity of the model. The more hidden units, the more parameters the model has. For instance, an MFNN having two hidden units is more complex than an MFNN with just one hidden unit, just as a second order polynomial is more complex than a first-order polynomial.

The problem is to calculate the model weights $\left(\mathrm{w}_{\mathrm{i}}\right)$ such that the input values are mapped to the output values. The weight calculation is also called model training. Since we force the model to learn the (observed) output values through the training procedure, the network training is called supervised. To estimate the model parameters, a cost function has to be minimized just like the LS procedure in Figure 2 (Bishop, 1995, pp.194-201). The difference is that, the closed 
solution of B in Figure 2 does not apply to MFNNs. We have to estimate the model parameters iteratively. The most effective iterative training technique is the Backpropagation (Rumelhart, Hilton, \& Williams, 1986). This is a method based on calculating the gradient of the cost function step-by-step. For each step, the gradient is used to update the parameters found in the previous step. The algorithm stops when satisfactory conditions have been met. It is important to note that, the hidden neurons play a primary role here. In fact, their output can be considered as a representation of the input in mapping the output (Rumelhart, Hilton, \& Williams, 1986). This property will be used for implementing Auto-Associative Neural Networks.

\section{Auto-associative Multi-layer Feed-forward Neural Networks}

An Auto-Associative Neural Network (AANN) is a particular kind of MFNN. Figure 6 shows an example of its topology.

Figure 6. An auto-associative multi-layer feed-forward neural network for non-linear dimensionality reduction.

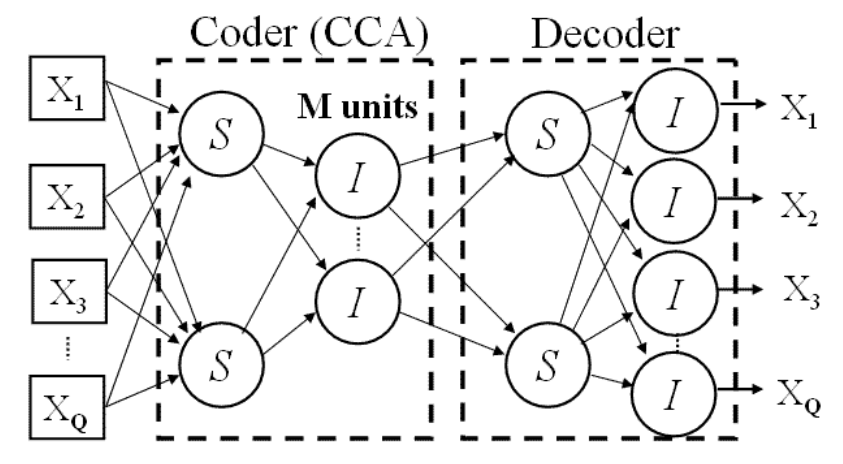

Inputs

Inputs

The aim of this kind of neural network is to perform an input dimensionality reduction in a nonlinear way. The strategy is based on mapping $Q$ input variables into $Q$ output variables. The observed outputs used to train the network (targets) are just the observed inputs themselves (for this reason this network is called auto-associative). The AANN in Figure 6 maps each observed input into itself (Bishop, 1995, p. 314). This strategy can be effectively used for dimensionality reduction if the number of the neurons in the second hidden layer (i.e., $M$ in Figure 6) is less than Q. So, the sub-network that we call Coder performs a compression of the Q inputs implementing the CCA. The sub-network that we call Decoder assures that the compression performed by the Coder is consistent with the input data.

To perform a correct non-linear dimensionality reduction, both units in the second hidden layer and the output units must be linear ( $\mathrm{I}=$ Identity function). Units in the first and third hidden layers must be non-linear ( $\mathrm{S}=$ Sigmoidal function). The training of this kind of network is based on minimizing a cost function similar to the one used for MFNNs (Bishop, 1995, p. 314).

Note that, the Coder projects the data from a Q-dimensional space into an M-dimensional space, while the Decoder performs the inverse mapping back to the Q-dimensional space. Curvilinear components are encapsulated in the Coder. This means that, once the AANN has been calibrated, the Coder can be used for transforming any input into an equivalent representation with respect to the original one exploiting fewer variables (from $\mathrm{Q}$ to $\mathrm{M}$ ). In other 
words, the Coder can execute a non-linear dimensionality reduction (CCA). This important result is made possible because of the presence of non-linear functions in the first and third hidden layer (S). This kind of AANN is able to perform also a linear dimensionality reduction as a particular case of the non-linear one.

\section{THE EMPIRICAL STRATEGY FOR MODEL IMPROVEMENT}

The aim of the model improvement strategy is to find empirically the most accurate model. Consider two distinct models $\mathrm{EM}_{1}$ and $\mathrm{EM}_{2}$ being compared. As discussed in the Section "Estimation Model Accuracy", we can argue that $\mathrm{EM}_{1}$ is more accurate (correct) than $\mathrm{EM}_{2}$ if two conditions are satisfied at the same time: (1) the bias of $\mathrm{EM}_{1}$ is closer to zero than the bias of $\mathrm{EM}_{2}$ and (2) the spread of $\mathrm{EM}_{1}$ is not worse than the spread of $\mathrm{EM}_{2}$. The problem is that, CCA can be applied with different reduction rates, i.e. with respect to Figure 6, the number of the units in the second hidden layer ( $\mathrm{M}$ ) may be set to $\mathrm{M}=\mathrm{Q}$ through to 1 , where $\mathrm{M}=\mathrm{Q}$ means no reduction and $\mathrm{M}=1$ means that the shrunken input data is expressed by only one component. But, we do not know the best CCA reduction rate (M), i.e. the one that bears the most accurate estimation model. To find it, we use an empirical strategy reported below (Sarcia', Cantone, \& Basili, 2008). The authors show that the strategy together with CCA can significantly improve accuracy of log-linear regression models calibrated on the COCOMO 81 data set (Shirabad \& Menzies, 2005). The strategy is the following:

\section{Precondition}

- Rely on a data set (DS) of past observations where each of them is expressed by $\mathrm{Q}$ independent variables $\mathrm{x}_{1}, \mathrm{x}_{2} \ldots \mathrm{x}_{\mathrm{Q}}$ and the $\mathrm{DS}$ size is $\mathrm{N}$ (i.e. $\left.\mathrm{DS}_{\mathrm{QXN}}\right)$ with $\mathrm{N}(=\mathrm{TR}+\mathrm{TE})$ statistically significant

- Independent variables $\mathrm{x}_{1}, \mathrm{x}_{2} \ldots \mathrm{x}_{\mathrm{Q}}$ are relevant to predict the dependent variable $\mathrm{y}$ and constitute the complete input set (Dreyfus, 2005, pp. 95-96).

Procedure

1. Split up $\mathrm{DS}_{\mathrm{QxN}}$ into two subsets (training and test set) as explained in Figure 3, obtaining $\mathrm{TRS}=\left(\mathrm{X}^{\prime}, \mathrm{Y}^{\prime}\right)$ and TES $=(\mathrm{X}, \mathrm{Y})($ Figure 7$)$

Figure 7. Data management for the empirical strategy

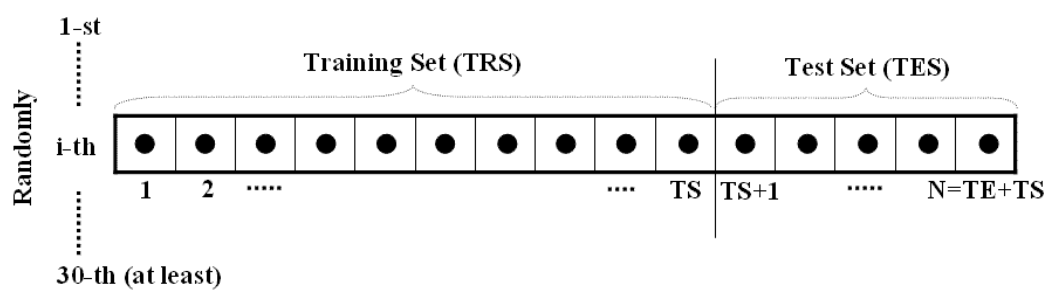

Note that, each dot in the array represents a data point (an observation belonging to $\mathrm{DS}_{\mathrm{QxN}}$ )

2. Use TRS (i-th row in Figure 7) to train $Q-1$ models (e.g. a log-linear regression function) by applying CCA as many times, where each time TRS is reduced by 1 component through 1 starting from $\mathrm{Q}-1$ components, i.e. in the first CCA application $\mathrm{M}=\mathrm{Q}-1$, in the second one $\mathrm{M}=\mathrm{Q}-2$ and so on up to $\mathrm{M}=1$

3. For each of the $\mathrm{Q}-1$ models in step 2, calculate the Mean $\left(\mathrm{RE}_{\mathrm{TE}}\right)$ and $\mathrm{STD}\left(\mathrm{RE}_{\mathrm{TE}}\right)$ as explained in Figure 3, i.e. feed TES into each of the $\mathrm{Q}-1$ models 
4. Based on the $\mathrm{Q}-1$ models obtained in step 2, select the model having the best Mean $\left(\mathrm{RE}_{\mathrm{TE}}\right)$ i.e., the closest to zero

5. Use TRS to train a model without applying CCA (labeled NO-CCA hereafter), i.e. a model without reduction

6. Calculate the Mean $\left(\mathrm{RE}_{\mathrm{TE}}\right)$ and $\mathrm{STD}\left(\mathrm{RE}_{\mathrm{TE}}\right)$ feeding TES into the NO-CCA model (step 5)

7. Repeat Steps 1 through 6 for a statistically sufficient number of times (e.g. 30) changing randomly the composition of TRS and TES (Figure 7) and get two samples (and so two distributions) for each considered summary statistic (Table 1), i.e., $\mathrm{MnRE}_{\mathrm{CCA}} \equiv$ $\left\{\operatorname{Mean}_{\mathrm{CCA}}\left(\mathrm{RE}_{\mathrm{TE}}^{(1)}\right) \ldots \operatorname{Mean}_{\mathrm{CCA}}\left(\mathrm{RE}_{\mathrm{TE}}^{(30)}\right)\right\}, \mathrm{STDRE}_{\mathrm{CCA}} \equiv\left\{\operatorname{STD}_{\mathrm{CCA}}\left(\mathrm{RE}_{\mathrm{TE}}{ }^{(1)}\right) \ldots\right.$ $\left.\operatorname{STD}_{\mathrm{CCA}}\left(\mathrm{RE}_{\mathrm{TE}}{ }^{(30)}\right)\right\}$, and MnRE $\mathrm{NO}_{\mathrm{CCA}} \equiv\left\{\operatorname{Mean}_{\mathrm{NO}-\mathrm{CCA}}\left(\mathrm{RE}_{\mathrm{TE}}{ }^{(1)}\right) \ldots \operatorname{Mean}_{\mathrm{NO}-\mathrm{CCA}}\left(\mathrm{RE}_{\mathrm{TE}}{ }^{(30)}\right)\right\}$, $\mathrm{STDRE}_{\mathrm{NO}-\mathrm{CCA}} \equiv\left\{\operatorname{STD}_{\mathrm{NO}-\mathrm{CCA}}\left(\mathrm{RE}_{\mathrm{TE}}{ }^{(1)}\right) \ldots \mathrm{STD}_{\mathrm{NO}-\mathrm{CCA}}\left(\mathrm{RE}_{\mathrm{TE}}{ }^{(30)}\right)\right\}$, respectively

\section{Table 1. Samples of statistics obtained by randomization}

\begin{tabular}{cccc}
\hline $\mathrm{MnRE}_{\mathrm{CCA}}$ & $\mathrm{STDRE}_{\mathrm{CCA}}$ & $\mathrm{MnRE}_{\mathrm{NO}-\mathrm{CCA}}$ & $\mathrm{STDRE}_{\mathrm{NO}-\mathrm{CCA}}$ \\
\hline $\operatorname{Mean}_{\mathrm{CCA}}\left(\mathrm{RE}_{\mathrm{TE}}{ }^{(1)}\right)$ & $\mathrm{STD}_{\mathrm{CCA}}\left(\mathrm{RE}_{\mathrm{TE}}{ }^{(1)}\right)$ & $\mathrm{Mean}_{\mathrm{NO}-\mathrm{CCA}}\left(\mathrm{RE}_{\mathrm{TE}}{ }^{(1)}\right)$ & $\mathrm{STD}_{\mathrm{NO}-\mathrm{CCA}}\left(\mathrm{RE}_{\mathrm{TE}}{ }^{(1)}\right)$ \\
$\mathrm{Mean}_{\mathrm{CCA}}\left(\mathrm{RE}_{\mathrm{TE}}{ }^{(2)}\right)$ & $\mathrm{STD}_{\mathrm{CCA}}\left(\mathrm{RE}_{\mathrm{TE}}{ }^{(2)}\right)$ & $\mathrm{Mean}_{\mathrm{NO}-\mathrm{CCA}}\left(\mathrm{RE}_{\mathrm{TE}}{ }^{(2)}\right)$ & $\mathrm{STD}_{\mathrm{NO}-\mathrm{CCA}}\left(\mathrm{RE}_{\mathrm{TE}}{ }^{(2)}\right)$ \\
$\vdots$ & $\vdots$ & $\vdots$ & $\vdots$ \\
$\operatorname{Mean}_{\mathrm{CCA}}\left(\mathrm{RE}_{\mathrm{TE}}{ }^{(30)}\right)$ & $\mathrm{STD}_{\mathrm{CCA}}\left(\mathrm{RE}_{\mathrm{TE}}{ }^{(30)}\right)$ & $\mathrm{Mean}_{\mathrm{NO}-\mathrm{CCA}}\left(\mathrm{RE}_{\mathrm{TE}}{ }^{(30)}\right)$ & $\mathrm{STD}_{\mathrm{NO}-\mathrm{CCA}}\left(\mathrm{RE}_{\mathrm{TE}}{ }^{(30)}\right)$ \\
\hline
\end{tabular}

8. Based upon suitable statistical tests (i.e., parametric or non-parametric), evaluate the hypotheses whether (1) $\mathrm{MnRE}_{\mathrm{CCA}}$ is significantly better than $\mathrm{MnRE}_{\mathrm{NO}-\mathrm{CCA}}$ and (2)

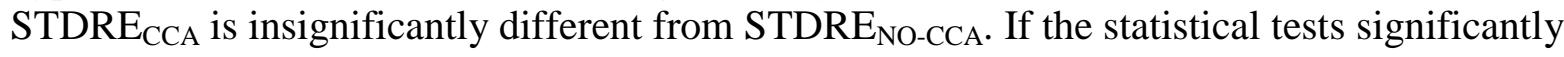
confirm hypotheses (1) and (2) at the same time, then execute steps 9 and 10, otherwise stop this procedure because CCA cannot significantly improve the accuracy. In the latter case, we can conclude that the data set $\mathrm{DS}_{\mathrm{NxQ}}$ has no curvilinear redundancy hence any compression will make worse accuracy of the model

9. Select the model corresponding to the best value in $\mathrm{MnRE}_{\mathrm{CCA}} \equiv\left\{\operatorname{Mean}_{\mathrm{CCA}}\left(\mathrm{RE}_{\mathrm{TE}}{ }^{(1)}\right) \ldots\right.$ $\left.\operatorname{Mean}_{\mathrm{CCA}}\left(\mathrm{RE}_{\mathrm{TE}}{ }^{(30)}\right)\right\}$. If two models have the same bias choose the one having the smallest spread; if two models have both the same bias and spread, choose one of them randomly 10. Use the chosen model for prediction.

\section{FUTURE RESEARCH DIRECTIONS}

The application of this strategy to the COCOMO 81 data set (Sarcia', Cantone, \& Basili, 2008) was able to improve the accuracy of log-linear models by randomizing the COCOMO 81 data set and applying two treatments, i.e., CCA and NO-CCA, and checked the results of both distributions using the Mann-Whitney and the Signed rank tests. We believe this successful application of the strategy is an encouraging step for the use of computational intelligence techniques. It offers a new direction for supporting traditional approaches in dealing with estimation problems. To increase the confidence of the software engineering community in the use of such emerging technologies, scholars, researchers, and practitioners should focus on 
replicating past experiments and performing new investigations based on different settings, e.g., by changing contexts and variables of interest.

An important future area of research is to compare the proposed CCA strategy to stepwise feature selection techniques, as well as exploring the possibility of defining a new strategy that combines both techniques.

\section{CONCLUSION}

In this chapter, we have discussed some issues concerning parametric estimation models based on regression functions. We have shown that these functions are relevant for any kind of estimation methodology even for human-based judgment techniques (McConnell, 2006, pp. 105112). The problem of improving the performance of regression functions is a major issue for any software organization that aims at delivering their products on time, on budget, and with the required quality. To improve the performance of regression functions, we have presented an empirical strategy based on a shrinking technology called CCA and showed its implementation by auto-associative neural networks. We have discussed the reasons why CCA is able to increase accuracy (correctness) without worsening the spread (variability) of the model (Sarcia', Cantone, $\&$ Basili, 2008). We have focused on the possibility of CCA to make models more parsimonious.

Let us now consider the implications of the empirical strategy reported above. From a practical point of view, an advantage of applying CCA is that we do not need to know the relevance of each attribute being removed with respect to the considered context. This is an advantage because, if we do not know the relevance of the attributes being removed, as stepwise feature selection techniques do, we cannot improve the model making it more parsimonious (Chen, Mezies, Port, \& Boehm, 2005; Kohavi \& John, 1997). Moreover, CCA does not suffer from multicollinearity, which affects stepwise methods. Multicollinearity is a statistical effect based on the impossibility of separating the influence of two or more input variables on the output (Weisberg, 1985). CCA overcomes this problem by considering the simultaneous effect of every input variable by finding non-linear redundancy of input variables in predicting the output. A further advantage is that, CCA can be completely automated, i.e. it does not require any human interaction to be carried out.

A real application of the presented strategy, is reported in (Sarcia', Cantone, \& Basili, 2008), where the authors provide some suggestions as to experimental setting, data management, and statistical tests. The authors find that, with respect to the COCOMO 81 data set, the proposed strategy increases correctness without worsening the reliability of log-linear regression models. Note that, the authors not only compared the medians, but also the standard deviations of the two distributions (i.e., CCA and NO-CCA). As presented above, they applied two non-parametric tests (Mann-Whitney and Signed rank) because a preliminary normality test on both distributions showed that the distributions could not be considered as coming from a normal population. Further results were that, applying the proposed strategy reduces the occurrence of outliers. In other words, estimates obtained by models treated with CCA show fewer outliers than models treated without applying CCA. This means that, CCA can improve reliability in terms of outlier reduction even though correctness may not be improved. This is a valuable result for researchers and practitioners because they may just use the strategy to build models less prone to outliers.

The strategy has some drawbacks. For instance, it has been built on the assumption of having enough data to split up the data set into two subsets (TRS and TES). Conversely, if we do not have enough data, CCA would not be applicable. CCA is based on building specific multi-layer 
neural networks, which require some optimization techniques to reduce the training time (Hagan \& Menhaj, 1994).

\section{REFERENCES}

Barron, A. (1993). Universal approximation bounds for superposition of a sigmoidal function. IEEE Transaction on Information Theory, 39(1), 930-945.

Boehm, B. (1981). Software Engineering Economics. Upper Saddle River, New Jersey, USA: Prentice-Hall.

Boehm, B., Horowitz, E., Madachy, R., Reifer, D., Clark, B.K., Steece, B., Brown, A.W., Chulani, S., \& Abts, C. (2000). Software Cost Estimation with COCOMO II. Upper Saddle River, New Jersey, USA: Prentice-Hall.

Bishop, C. (1995). Neural Network for Pattern Recognition. New York, NY, USA: Oxford University Press.

Chen, Z. Mezies, T. Port, D., \& Boehm, B.W. (2005). Feature Subset Selection Can Improve Software Cost Estimation Accuracy. In T. Mezies (Ed.), PROMISE ’05 (pp. 1-6). ACM.

Conte, S.D., Dunsmore, H.E., \& Shen, V.Y. (1986). Software Engineering Metrics and Models. Menlo Park, CA, USA: The Benjamin/Cummings Publishing Company, Inc.

Dreyfus, G. (2005). Neural Networks Methodology and Applications. Berlin, Germany: Springer.

Hagan, M.T., \& Menhaj, M.B. (1994). Training Feedforward Networks with the Marquardt Algorithm. IEEE Transactions on Neural Networks. 5(6), 989-993.

Jollife, I.T. (1986). Principal Component Analysis. Berlin, Germany: Springer.

Kitchenham, B., MacDonell, S., Pickard, L., \& Shepperd, M. (2001). What accuracy statistics really measure. IEE Proceedings: Vol. 148. Software Engineering (pp. 81-85). IEE Proceeding.

Kohavi, R., \& John, G.H. (1997). Wrappers for feature subset selection. ACM Artificial Intelligence, 97(1-2), 273-324.

McConnell, S. (2006). Software Estimation - Demystifying the Black Art. Redmond, WA, USA: Microsoft press.

McQuarrie, A.D.R., \& Tsai, C. (1998). Regression and Time Series Model Selection. Singapore: World Scientific Publishing Co. Pte. Ltd..

Myrtveit, I., Stensrud, E., \& Shepperd, M. (2005). Reliability and Validity in Comparative Studies of Software Prediction Models. IEEE Transaction on Software Engineering, 31(5), 380391. 
Pedhazur, E.J. (1997). Multiple Regression in Behavioral Research. Orlando, FL, USA: Harcourt Brace.

Rumelhart, D.E., Hilton, G.E., \& Williams, R.J. (1986). Learning Internal Representations by Error Propagation. In D. Rumelhart \& J. McClelland (Ed.): Parallel Distributing Computing: Explorations in the Microstructure of Cognition: Vol. 1. (pp. 318-362). The MIT press.

Sarcia', S.A., Cantone, G, \& Basili, V.R (2008). Adopting Curvilinear Component Analysis to Improve Software Cost Estimation Accuracy. Model, Application Strategy, and an Experimental Verification. In G. Visaggio (Ed.), $12^{\text {th }}$ International Conference on Evaluation and Assessment in Software Engineering. BCS eWIC.

Shepperd, M. (2007). Software project economics: a roadmap. International Conference on Software Engineering 2007: Vol. 1. IEEE Future of Software Engineering (pp. 304-315). IEEE Computer Society

Shirabad, J.S., \& Menzies, T. (2005). The PROMISE Repository of Software Engineering Databases. School of Information Technology and Engineering of the University of Ottawa, Canada. Retrieved August 31, 2008, from http://promise.site.uottawa.ca/SERepository.

Weisberg, S. (1985). Applied Linear Regression, New York, NY, USA: John Wiley and Sons.

\section{ADDITIONAL READING}

Aha, D.W., \& Bankert, R.L. (1996). A comparative evaluation of sequential feature selection algorithms. Artificial Intelligence and Statistics. New York, NY, USA: Springer-Verlag.

Angelis, L., \& Stamelos, I. (2000). A Simulation Tool for efficient analogy based cost estimation," Empirical Software Engineering, 5(1), 35-68.

Basili, V.R., \& Weiss, D. (1984). A Methodology for Collecting Valid Software Engineering Data. IEEE Transactions on Software Engineering, 3(1), 728-738.

Bishop, C., \& Qazaz, C.S. (1995). Bayesian inference of noise levels in regression. International Conference on Artificial Neural Networks: Vol. 2. EC2 \& Cie (pp. 59-64). ICANN95.

Briand, L.C., Basili, V.R. \& Thomas, W. (1992). A pattern recognition approach to software engineering data analysis. IEEE Transactions on Software Engineering. 18(11), 931-942, IEEE Computer Society.

Briand, L.C., El-Emam, K., Maxwell, K., Surmann, D., \& Wieczorek, I. (1999). An Assessment and Comparison of Common Cost Software Project Estimation Methods. Proceeding of the 1999 International Conference on Software Engineering (pp. 313-322): IEEE Computer Society. 
Briand, L.C., Langley, T., \& Wieczorek, I. (2000). A Replicated Assessment and Comparison of Common Software Cost Modeling Techniques. Proceeding of the 2000 International Conference on Software Engineering (pp. 377-386). IEEE Computer Society.

Cantone, G., \& Donzelli, P. (2000). Production and Maintenance of Goal-oriented Measurement Models. World Scientific, 105(4), 605-626.

Fenton, N. E. (1991). Software Metrics: A Rigorous Approach. London, UK: Chapman \& Hall.

Finnie, G., Wittig, G., \& Desharnais, J.-M. (1997). A comparison of software effort estimation techniques using function points with neural networks, case based reasoning and regression models. Journal of Systems \& Software. 39(3), 281-289.

Foss, T., Stensrud, E., Kitchenham, B., \& Myrtveit, I. (2003). A Simulation Study of the Model Evaluation Criterion MMRE. IEEE Transaction on Software Eng. 29(11), 985-995.

Gulezian, R. (1991). Reformulating and calibrating COCOMO. Journal of Systems \& Software. 16(6), 235-242.

Guyon, I, Gunn, M., Nikravesh, \& Zadeh, L. (2005). Feature Extraction foundations and applications. Berlin, Germany: Springer.

Jeffery, R., \& Low, G. (1990). Calibrating estimation tools for software development. Software Engineering Journal. 5(4), 215-221.

John, G., Kohavi, R., \& Pfleger, K. (1994). Irrelevant features and the subset selection problem. 11th Intl. Conference on Machine Learning (pp. 121-129). Morgan Kaufmann.

Kemerer, C.F., (1987). An Empirical Validation of Software Cost Estimation Models. ACM Communications, 30(5), 416-429.

Khoshgoftaar, T. M., \& Lanning, D. L. (1995). A Neural Network Approach for Early Detection of Program Modules Having High Risk in the Maintenance Phase. Journal of Systems Software. 29(1), 85-91.

Khoshgoftaar, T. M., Lanning, D. L., \& Pandya, A. S. (1994). A comparative-study of patternrecognition techniques for quality evaluation of telecommunications software. IEEE Journal on Selected Areas In Communications, 12(2), 279-291.

Kirsopp, C. \& Shepperd, M. (2002). Case and Feature Subset Selection in Case-Based Software Project Effort Prediction. Research and Development in Intelligent Systems XIX, New York, NY, USA: Springer-Verlag.

Menzies, T., Port, D., Chen, Z., \& Hihn, J. (2005). Validation Methods for Calibrating Software Effort Models. Proceeding of the 2000 International Conference on Software Engineering (587595). IEEE Computer Society. 
Myrtveit, I., \& Stensrud, E. (1999). A controlled experiment to assess the benefits of estimating with analogy and regression models. IEEE Transaction on Software Engineering, 25(4), 510525.

Myrtveit, I., \& Stensrud, E. (2004). Do Arbitrary Function Approximators make sense as Software Prediction Models? 12-th International Workshop on Software Technology and Engineering Practice (pp. 3-9). IEEE Computer Society.

Neumann, D.E. (2002). An Enhanced Neural Network Technique for Software Risk Analysis. IEEE Transaction on Software Engineering, 28(9), 904-912.

Rao, C.R. (1973). Linear Statistical Inference and its Applications. New York, NY, USA: Wiley $\&$ Sons.

Srinivasan, K., \& Fisher, D. (1995). Machine Learning Approaches to Estimating Software Development Effort. IEEE Transaction on Software Engineering, 21(2), 126-137.

Stensrud, E., Foss, T., Kitchenham, B., \& Myrtveit, I. (2002). An empirical Validation of the Relationship between the Magnitude of Relative Error and Project Size. Proceeding of the 8-th IEEE Symposium on Software Metrics (pp. 3-12). IEEE Computer Society.

Vapnik, V.N. (1995). The Nature of Statistical Learning Theory. New York, NY, USA: Springer-Verlag.

Wohlin, C., Runeson, P., Höst, M., Ohlsson, M.C., Regnell, B., \& Wesslén, A. (2000). Experimentation in Software Engineering - An Introduction. Berlin, Germany: Springer.

Salvatore Alessandro Sarcia' received his laurea degree in Computer Science (1994) and master degree with honors in Strategy and Project Management (2001) from the University of Torino (Italy). He received his second laurea degree in Politics (2004) from the University of Trieste (Italy). In December 2008, he received his Ph.D. degree in Informatics and Automation Engineering from the University of Rome "Tor Vergata" (Italy). In 2006-08, he was with the Department of Computer Science of the University of Maryland (College Park, MD, USA) as a Faculty Research Assistant. Mr. Sarcia' is an untenured Professor of Object-Oriented Programming at the DISP - University of Rome "Tor Vergata". His research interests lay in the area of Computational Intelligence (Artificial Neural Networks), Predictive Models, and Statistics. He applies empirical techniques to conduct experiments on Software Engineering, Software Quality, and Risk Analysis.

Dr. Salvatore Alessandro Sarcia' DISP - University of Rome "Tor Vergata"

Via del Politecnico, 1

00133 Rome (Italy)

Voice: +39.06.7259.7942, Fax: +39.06.7259.7460 
http://www.onese.org/sarcia

sarcia@disp.uniroma2.it

Victor R. Basili is Professor Emeritus at the University of Maryland. He

holds a PH.D. in Computer Science from the University of Texas and two honorary degrees. He was Director of the Fraunhofer Center - Maryland and a director of the Software Engineering Laboratory at NASA/GSFC. He has worked on measuring, evaluating, and improving the software development process and product for over 35 years with numerous companies and government agencies. Methods include Iterative Enhancement, the Goal Question Metric Approach (GQM), the Quality Improvement paradigm (QIP), and the Experience Factory (EF).

Dr. Basili has authored over 250 peer reviewed publications and is a recipient of several awards including the NASA Group Achievement Awards, ACM SIGSOFT Outstanding Research Award, IEEE Computer Society Harlan Mills Award, and the Fraunhofer Medal. He is Co-EIC of the Springer Empirical software Engineering Journal and an IEEE and ACM Fellow.

Professor Victor Robert Basili

Department of Computer Science and Institute for Advanced Computer Studies University of Maryland

College Park, Maryland 20742

Phone: 301-405-2668 Fax: 301-405-3691

http://www.cs.umd.edu/ basili/

basili@cs.umd.edu

and

Fraunhofer Center for Experimental Software Engineering - Maryland

College Park Maryland 20742

Phone: 301-403-8934 Fax:301-403-8976

http://fc-md.umd.edu

Professor Giovanni Cantone is Professor at the University of Rome "Tor Vergata" teaching Software Analysis, Design, and Architectures.

Professor Giovanni Cantone

DISP - University of Rome "Tor Vergata"

Via del Politecnico, 1

00133 Rome (Italy)

Voice: +39.06.7259.7392, Fax: +39.06.7259.7460

cantone@uniroma2.it 